\title{
Avaliação agronômica e identificação de quimiotipos de erva cidreira no Distrito Federal
}

\author{
Hermes Jannuzzi ${ }^{1}$; Jean Kleber A Mattos'; Roberto F Vieira²; Dijalma B Silva² ${ }^{2}$;umberto R Bizzo ${ }^{3}$; \\ Ligia AM Gracindo ${ }^{2}$ \\ ${ }^{1}$ UnB-Fac. Agronomia e Medicina Veterinária, C. Postal 4508, 70910-970 Brasília-DF; 22Embrapa Recursos Genéticos e Biotecnologia, \\ C. Postal 2372, 70770-900 Brasília-DF; ${ }^{3}$ Embrapa Agroindústria de Alimentos, Av. das Américas, 29501, 23020-470 Rio de Janeiro- \\ RJ; jhermes@brturbo.com.br; kleber@unb.br; rfvieira@cenargen.embrapa.br; dijalma@cenargen.embrapa.br; bizzo@ctaa.embrapa.br; \\ ligia@cenargen.embrapa.br
}

\section{RESUMO}

Com o objetivo de avaliar o comportamento agronômico, o potencial de rendimento de óleo essencial e identificar os quimiotipos de 16 acessos de Lippa alba (erva cidreira) da coleção da Universidade de Brasília foi conduzido um experimento de campo em Latossolo Vermelho, sob irrigação por gotejamento na região do Cerrado do Distrito Federal. Foram avaliados a época de florescimento, hábito de crescimento, área foliar, comprimento da haste, massa fresca de folhas e hastes, massa foliar seca, teor e rendimento de óleo essencial e perfil aromático. $\mathrm{O}$ óleo essencial foi extraído por hidrodestilação em aparelho de Clevenger modificado e os constituintes identificados e quantificados através de cromatografia em fase gasosa acoplada a espectrometria de massas. O delineamento experimental utilizado foi de blocos ao acaso, com três repetições, contendo três plantas úteis por parcela. Foram identificados os quimiotipos citral-limoneno, citral-mirceno, limoneno-carvona, citral, linalol, mirceno e linalollimoneno. Os acessos apresentaram teores máximos de linalol de 89,8\% (L.16); mirceno de 47,6\% (L.37); limoneno de 36,0\%, carvona de $46,9 \%$ (L.27) e citral de $56,7 \%$ (L.17). Os genótipos com maiores áreas foliares e maiores comprimentos de hastes tenderam a apresentar maiores teores de óleo e maior concentração de linalol. A concentração de óleo foi inversamente proporcional à produção de massa foliar seca. Os acessos que apresentaram os maiores rendimentos dos compostos majoritários do óleo essencial foram: L.16 $\left(0,77 \mathrm{~g} \mathrm{pl}^{-1}\right)$, como fonte potencial para a produção de linalol; L.17 $\left(0,17 \mathrm{~g} \mathrm{pl}^{-1}\right)$, como fonte de mirceno; L. $27\left(0,17 \mathrm{~g} \mathrm{pl}^{-1}\right)$, como fonte de limoneno e de carvona $\left(0,21 \mathrm{~g} \mathrm{pl}^{-1}\right)$ e L.38 $\left(0,24 \mathrm{~g} \mathrm{pl}^{-1}\right)$, como fonte de citral.

Palavras-chave: Lippia alba, óleo essencial, limoneno, linalol, mirceno, citral.

\begin{abstract}
Agronomic evaluation and identification of Lippia alba chemotypes from Distrito Federal, Brazil
\end{abstract}

The main objective of this work was to describe the essential oil profile of 16 genotypes of Lippia alba from the germplasm collection of the Universidade de Brasília and to analyze its potential of production. A field assay was carried out in the rural area of Distrito Federal and the following parameters were analyzed: flowering period, growing habit, foliar area, length of the main branch, fresh and dry weight of the biomass (leaves and branches), essential oil content and profile of the constituent volatile oils. Essential oil was extracted using a modified Clevenger apparatus and the constituent volatile oils were analyzed by gas chromatography and GC/MS. The experimental design was randomized blocks with three plants per plot. The following chemotypes were reported: citral-limonene, citral-myrcene, limonene-carvone, citral, linalool, myrcene and linalool-limonene. Higher levels of linalool were found in genotype L. 16 (89.8\%); myrcene in L.37 (47.6\%); limonene in L. 27 (36.0\%); carvone in L.27 (46.9\%) and citral in L.17 (56.7\%). The genotypes with the highest leaf area and leaf length of the main branch seem to be correlated with the best yield of essential oil and the higher level of linalool. The yield of essential oil was inversely proportional to the dry weight of biomass. The accessions which presented the highest average yield of the major essential constituent oils were: L.16(0.77 $\left.\mathrm{g} \mathrm{pl}^{-1}\right)$ as a source of raw material for linalool production; L.17 $(0.17$ $\left.\mathrm{g} \mathrm{pl}^{-1}\right)$ as source of myrcene; L. 27 as source of limonene $\left(0.17 \mathrm{~g} \mathrm{pl}^{-1}\right)$ and carvone $\left(0.21 \mathrm{~g} \mathrm{pl}^{-1}\right)$; and L.38 $\left(0.24 \mathrm{~g} \mathrm{pl}^{-1}\right)$ as a citral source.

Keywords: Lippia alba, essential oil, limonene, linalool, myrcene, citral.

(Recebido para publicação em 19 de fevereiro de 2009; aceito em 27 de setembro de 2010) (Received on February 19, 2009; accepted on September 27, 2010)

\begin{abstract}
$\mathrm{A}^{\mathrm{c}}$ erva cidreira (Lippia alba, Verbenaceae) é uma planta bastante utilizada pela ampla variabilidade química dos seus óleos essenciais. Essa diversificação de componentes do óleo essencial é responsável por suas várias utilizações na medicina popular e na fitoterapia. Os óleos essenciais contidos em suas folhas possuem propriedades antibacterianas e antifúngicas (Alea $e t$ al., 1997; Matos, 1996; Soares, 2001; Pessini et al., 2003).
\end{abstract}

Dentre os componentes do óleo essencial de $L$. alba, o linalol tem se destacado pelo seu expressivo rendimento na planta, por sua ampla utilização nas indústrias de aromatizantes, cosméticos e perfumes (Ehlert, 2003), e pelas suas propriedades inseticidas, antiinflamatórias e anti-sépticas (Simões \& Spitzer, 2003).

Lorenzi \& Matos (2002) sugerem a condução de novos estudos com L. alba considerando os aspectos fitotécnicos com a identificação de quimiotipos, visando garantir a produção de matériaprima em quantidade e qualidade para a elaboração de produtos fitoterápicos eficazes e seguros. Devido à falta de um padrão definido para a distinção de quimiotipos em $L$. alba, a maioria dos autores tem se baseado na relação entre os compostos majoritários de forma particular. Desta forma, Matos (1996) identificou três quimiotipos para as ervas cidreiras do nordeste: citral-mirceno, 
citral-limoneno, e limoneno-carvona. Siani et al. (2002) e Santos-Mendes et al. (2000) citados por Gilbert et al. (2005) identificaram os quimiotipos linalol e citral.

A maioria dos estudos com plantas medicinais no Brasil tem sido realizada com plantas exóticas e, quando se trata de plantas nativas, estes trabalhos, além de escassos, têm sido pouco abrangentes. A carência de informações agronômicas sobre plantas medicinais não é um problema apenas no Brasil, mas também em outros países. A falta de dados agronômicos associados ao rendimento de metabólitos secundários, principalmente óleo essencial de L. alba constitui-se em uma limitação para o estabelecimento de sistemas de cultivos comerciais.

O objetivo deste trabalho foi avaliar o comportamento agronômico, o potencial de rendimento de óleo essencial e identificar os quimiotipos de 16 acessos de L. alba da coleção da Universidade de Brasília, na região do Distrito Federal.

\section{MATERIAL E MÉTODOS}

Foram avaliados 16 acessos da coleção de L. alba da UnB, a seguir relacionados: L.1 (Loteamento Rural"ABC"-DF); L.2 (Araguaina-TO); L.4 (São Gonçalo do Rio Abaixo-MG); L.5 (Botucatu-SP); L.16 (Loteamento urbano Estrutural-DF); L.17 (Florianópolis-SC); L.20 (Goiânia-GO); L.22 (Ilhéus-BA); L.24 (Lavras-MG); L.25 (Luminárias-MG); L.27 (ManausAM); L.28 (Muzambinho-MG); L.29 (Piracicaba-SP); L.31 (Planaltina de Goiás-GO); L.37 (São Paulo-SP) e L.38 (SQN205-DF). As exsicatas dos acessos foram depositadas no Herbário da Embrapa Recursos Genéticos e Biotecnologia (Cenargen) e sua identificação botânica foi estabelecida pelo Dra. Fátima Salimento, especialista na família Verbenaceae.

O experimento foi conduzido na Chácara “Chão do Cafuringa" (15³7'05”'S; 48 03'46”'W; $1.283 \mathrm{~m}$ de altitude), em processo de certificação orgânica, clima Köppen Cwb, e latossolo vermelho em toda a extensão da área do experimento. A análise química de solo na camada de 0 a 20 cm indicou: $\mathrm{pH}=5,9 ; \mathrm{Ca}=3,4$ meq $100 \mathrm{~mL}^{-1} ; \mathrm{Mg}=0,7$ meq $100 \mathrm{~mL}^{-1}$;
$\mathrm{K}=0,04$ meq $100 \mathrm{~mL}^{-1} ; \mathrm{Na}=0,01 \mathrm{meq}$ $100 \mathrm{~mL}^{-1}, \mathrm{P}=1 \mathrm{ppm} ; \mathrm{Al}=0,1 \mathrm{meq} 100$ $\mathrm{mL}^{-1}$; CTC a pH 7=8,8 meq $100 \mathrm{~mL}^{-1}$; saturação por base $=47 \%$; matéria orgânica $=12,0 \mathrm{~g} \mathrm{~kg}^{-1} ; \mathrm{B}=0,04 \mathrm{mg} \mathrm{dm}^{-3} ; \mathrm{Cu}=$ $0,45 \mathrm{mg} \mathrm{dm}^{-3} ; \mathrm{Fe}=81,9 \mathrm{mg} \mathrm{dm}^{-3} ; \mathrm{Mn}=$ $13,0 \mathrm{mg} \mathrm{dm}^{-3} ; \mathrm{Zn}=6,38 \mathrm{mg} \mathrm{dm}^{-3}$ e $\mathrm{S}=$ $5,7 \mathrm{mg} \mathrm{dm}^{-3}$. A análise física identificou granulometria $=375 \mathrm{~g} \mathrm{~kg}^{-1}$, silte $=275 \mathrm{~g}$ $\mathrm{kg}^{-1}$ e argila $=350 \mathrm{~g} \mathrm{~kg}^{-1}$.

As mudas foram produzidas por estaquia em casa de vegetação e aclimatadas por 10 dias antes do plantio na área experimental. $\mathrm{O}$ plantio foi realizado em 19 de fevereiro em solo arado e gradeado, utilizando espaçamento de 2,0 m entre linhas e 2,0 m entre plantas, covas de $0,4 \times 0,4 \times 0,4 \mathrm{~m}$ adubadas com $500 \mathrm{~g}$ de termofosfato Yorin, $20 \mathrm{~g}$ FTE (Fritted Trace Elements) e 2,0 L de húmus de minhoca. As plantas receberam irrigação individual por gotejamento, (vazão média de 2,0 $\mathrm{L} \mathrm{h}^{-1}$ ) com turno de rega a cada três dias, por duas horas no período noturno.

Foram realizadas capinas manuais nas entrelinhas a cada 30 dias, até o mês de outubro quando foi realizada uma poda a $10 \mathrm{~cm}$ de altura do solo em todas as plantas e adubação em cobertura com $200 \mathrm{~g}$ de cama de frango (3\% de $\mathrm{N})$. A partir dessa data as capinas foram efetuadas a cada 25 dias. A colheita foi realizada entre 7 e 18 de março.

O delineamento experimental foi de blocos ao acaso, com três repetições, contendo três plantas por parcela, com arranjo simples.

Foram avaliados a época de florescimento, hábito de crescimento, área foliar, comprimento da haste, massa fresca na colheita (parte aérea, folhas e hastes), massa foliar seca, teor e perfil aromático do óleo essencial.

Foi considerada época de florescimento quando $50 \%$ das plantas apresentavam florescimento. O hábito de crescimento das plantas foi classificado em três categorias: a) decumbente (planta com ramos rastejante ao solo); b) prostrado (planta com crescimento inicial ereto e posteriormente curvado, podendo ou não tocar o solo) e c) ereto (planta com crescimento ereto ou vertical).

Para o calculo da área foliar $\left(\mathrm{cm}^{2}\right)$, foram coletadas as folhas do quinto nó dos dois maiores ramos de duas plantas de cada parcela. As folhas foram digitalizadas e as imagens analisadas e mensuradas pelo software Image Pro Plus, versão 4.5 para Windows. O comprimento das hastes na unidade metro $(\mathrm{m})$ foi determinado medindo-se as duas maiores hastes de três plantas, em cada parcela.

A massa fresca $\left(\mathrm{g} \mathrm{pl}^{-1}\right)$ na colheita foi obtida após o corte das plantas a $10 \mathrm{~cm}$ de altura do solo, no período da manhã. A massa foliar seca $\left(\mathrm{g} \mathrm{pl}^{-1}\right)$ foi obtida após, lavagem das plantas, retirada das hastes, secagem ao ar livre por $24 \mathrm{~h}$, derriça manual das folhas, secagem em estufa com circulação forçada de ar a $38^{\circ} \mathrm{C}$ até atingir peso constante, com pesagem em balança digital.

A extração do óleo essencial foi realizada pelo método de hidrodestilação em aparelho do tipo Clevenger modificado (Matos, 1996) utilizandose balão, com capacidade de 2 L onde foram colocadas $100 \mathrm{~g}$ de folhas secas, acrescidas de 1,5 L de água destilada por 90 minutos (Castro, 2001; Stashenko et al., 2003), com três repetições. O óleo foi coletado com micropipeta e colocado em vidro cor âmbar de $5 \mathrm{~mL}$. Após a pesagem o óleo foi conservado em geladeira $\mathrm{a}-5^{\circ} \mathrm{C}$ sob abrigo da luz, até o momento da sua análise química. O teor de óleo expresso em porcentagem foi obtido a partir da relação da massa do óleo extraído $(\mathrm{g})$ pela massa de folhas secas no balão (g), multiplicada por 100. $\mathrm{O}$ rendimento de óleo essencial $\left(\mathrm{g} \mathrm{pl}^{-1}\right)$ foi estimado a partir da relação entre o teor de óleo (\%) e a massa foliar seca de folha por planta.

$\mathrm{Na}$ avaliação do perfil aromático do óleo essencial das amostras de L. alba foi utilizado o cromatógrafo Shimadzu GC 17A com auto-injetor AOC-20i, em coluna capilar HP-5 (25 m x 0,32 mm $\mathrm{x} 0,25 \mu \mathrm{m})$, com temperatura de forno variando de 60 a $240^{\circ} \mathrm{C}$ a $3^{\circ} \mathrm{C} \mathrm{min}^{-1}$, e utilizando o hidrogênio como gás carreador $\left(1,4 \mathrm{~mL} \mathrm{~min}^{-1}\right)$. Foram injetados $0,05 \mu \mathrm{l}$ de óleo puro diluídos em $1,5 \mathrm{~mL}$ de diclorometano no modo com divisão de fluxo $\left(1: 100\right.$; injetor a $\left.250^{\circ} \mathrm{C}\right)$. A identificação dos constituintes químicos foi baseada nos índices de retenção (IR), calculados a partir do tempo de retenção dos componentes dos óleos e de uma 
série homóloga de n-alcanos injetados nas mesmas condições, e comparação dos espectros de massas obtidos com os dados da biblioteca Wiley $6^{\text {th }}$ ed. A identificação dos constituintes majoritários foi feita conforme descrita em Potzernheim et al. (2006). Para identificação dos quimiotipos foram considerados apenas os constituintes majoritários do óleo essencial com concentrações acima de $10 \%$.

Os dados experimentais foram submetidos à análise de variância e teste de médias (Scott-Knott 5\%) utilizando o aplicativo SISVAR 4.6 e análise de correlação de Pearson utilizando o aplicativo SAEG 5.0. O coeficiente de correlação foi interpretado de acordo com os critérios sugeridos por Shimakura \& Ribeiro Junior (2006) como a seguir: 0,00 a 0,19 (bem fraca); 0,20 a 0,39 (fraca); 0,40 a 0,69 (moderada); 0,70 a
0,89 (forte); 0,90 a 1,00 (muito forte).

\section{RESULTADOS E DISCUSSÃO}

A floração iniciou-se em meados de dezembro, com auge no mês de janeiro do ano seguinte, observando-se a visita constante de abelhas. Os acessos apresentaram hábito de crescimento variável, com combinações entre os três tipos: ereto, prostrado e decumbente (Tabela 1). Observou-se que além da herança genotípica, a variação no hábito de crescimento pode ter ocorrido em função do corte e da rebrota sob ação persistente dos ventos locais, que induziram ao arqueamento e quebra das hastes finas alterando o padrão de crescimento das plantas. Observou-se que as hastes tombadas quando em contato com o solo emitiam raízes e novas brotações.

Os acessos mostraram grande hete- rogeneidade em relação ao tamanho das folhas, podendo ser agrupados em duas categorias: uma com folhas grandes, com áreas foliares variando de 16 a 21 $\mathrm{cm}^{2}$ e outra com folhas menores com variação de 4,2 a 12,0 $\mathrm{cm}^{2}$ (Tabela 1). Analisando as médias das áreas foliares e o hábito de crescimento dos acessos, observou-se que os acessos com hábito "prostrado e decumbente" apresentam as maiores médias de área foliar, com exceção do acesso L.05.

Os acessos L.1, L.4, L.24, L.28 e L.29 apresentaram os maiores comprimentos de haste e hábito de crescimento prostrado e decumbente. Pereira Pinto et al. (2000) e Gilbert et al. (2005) citam que existem grandes variações no comprimento de hastes desta espécie.

A massa fresca variou de $3.463 \mathrm{~g} \mathrm{pl}^{-1}$ a $906 \mathrm{~g} \mathrm{pl}^{-1}$ com valor médio de 2.045 $\mathrm{g} \mathrm{pl}^{-1}$. Os acessos L.22 (3.463 $\mathrm{g} \mathrm{pl}^{-1}$ )

Tabela 1. Hábito de crescimento e valores médios de área foliar, comprimento da haste, massa fresca (parte aérea (folhas e haste)), massa seca (folhas), teor percentual de óleo essencial (OE) e rendimento de óleo essencial de acessos de $L$. alba [growing habit and average values of leaf area, length of the main branch, fresh weight of the biomass (leaves and branches), dry weight of leaves, essential oil content and yield of L. alba accessions]. Brasília, Universidade de Brasília, 2006.

\begin{tabular}{|c|c|c|c|c|c|c|c|}
\hline Acessos & $\begin{array}{l}\text { Hábito de cresci- } \\
\text { mento }\end{array}$ & $\begin{array}{l}\text { Área foliar } \\
\left(\mathrm{cm}^{2}\right)\end{array}$ & $\begin{array}{c}\text { Comprimento } \\
\text { da haste (m) }\end{array}$ & $\begin{array}{c}\text { Massa fresca } \\
\qquad\left(\mathrm{g} \mathrm{pl}^{-1}\right)\end{array}$ & $\begin{array}{l}\text { Massa foliar } \\
\text { seca }\left(\mathrm{g} \mathrm{pl}^{-1}\right)\end{array}$ & $\begin{array}{c}\text { Teor OE } \\
(\%)\end{array}$ & $\begin{array}{c}\text { Rendimento } \\
\mathrm{OE}\left(\mathrm{g} \mathrm{pl}^{-1}\right)\end{array}$ \\
\hline L.01 & $\begin{array}{l}\text { Prostrado e de- } \\
\text { cumbente }\end{array}$ & $16,5 \mathrm{a}$ & $2,7 \mathrm{a}$ & $2.230 \mathrm{c}$ & $99,4 \mathrm{~b}$ & $0,59 \mathrm{~b}$ & $0,60 \mathrm{a}$ \\
\hline L.02 & Ereto & $9,5 \mathrm{~b}$ & $1,9 \mathrm{~b}$ & $2.621 \mathrm{~b}$ & $94,6 \mathrm{~b}$ & $0,43 \mathrm{c}$ & $0,40 \mathrm{~b}$ \\
\hline L.04 & $\begin{array}{l}\text { Prostrado e de- } \\
\text { cumbente }\end{array}$ & $20,4 \mathrm{a}$ & $2,6 \mathrm{a}$ & $1.749 \mathrm{c}$ & $104,0 \mathrm{~b}$ & $0,62 \mathrm{~b}$ & $0,68 \mathrm{a}$ \\
\hline L. 05 & $\begin{array}{l}\text { Prostrado e de- } \\
\text { cumbente }\end{array}$ & $9,3 \mathrm{~b}$ & $1,8 \mathrm{~b}$ & $2.424 \mathrm{~b}$ & $90,3 \mathrm{~b}$ & $0,38 \mathrm{c}$ & $0,35 \mathrm{~b}$ \\
\hline L. 16 & $\begin{array}{l}\text { Ereto, prostrado e } \\
\text { decumbente }\end{array}$ & $16,0 \mathrm{a}$ & $1,8 \mathrm{~b}$ & $1.502 \mathrm{~d}$ & $63,6 \mathrm{c}$ & $0,84 \mathrm{a}$ & $0,53 \mathrm{a}$ \\
\hline L. 17 & Ereto & $10,2 \mathrm{~b}$ & $1,8 \mathrm{~b}$ & $2.046 \mathrm{c}$ & $86,6 \mathrm{~b}$ & $0,35 \mathrm{c}$ & $0,31 \mathrm{~b}$ \\
\hline L. 20 & Ereto e prostrado & $12,0 \mathrm{~b}$ & $1,7 \mathrm{~b}$ & $2.071 \mathrm{c}$ & $89,7 \mathrm{~b}$ & $0,28 \mathrm{~d}$ & $0,25 \mathrm{~b}$ \\
\hline L. 22 & Ereto e prostrado & $9,9 \mathrm{~b}$ & $1,7 \mathrm{~b}$ & $3.463 \mathrm{a}$ & $207,7 \mathrm{a}$ & $0,19 \mathrm{~d}$ & $0,40 \mathrm{~b}$ \\
\hline L. 24 & $\begin{array}{l}\text { Prostrado e de- } \\
\text { cumbente }\end{array}$ & $17,8 \mathrm{a}$ & $2,7 \mathrm{a}$ & $1.995 \mathrm{c}$ & $96,4 \mathrm{~b}$ & $0,76 \mathrm{a}$ & $0,73 \mathrm{a}$ \\
\hline L. 25 & $\begin{array}{l}\text { Prostrado e de- } \\
\text { cumbente }\end{array}$ & $16,8 \mathrm{a}$ & $2,5 \mathrm{~b}$ & $1.824 \mathrm{c}$ & $86,4 \mathrm{~b}$ & $0,64 \mathrm{c}$ & $0,54 \mathrm{a}$ \\
\hline L. 27 & Ereto e prostrado & $4,2 \mathrm{~b}$ & $1,7 \mathrm{~b}$ & $1.442 \mathrm{~d}$ & $70,0 \mathrm{c}$ & $0,69 \mathrm{~b}$ & $0,47 \mathrm{~b}$ \\
\hline L. 28 & $\begin{array}{l}\text { Prostrado e de- } \\
\text { cumbente }\end{array}$ & $16,1 \mathrm{a}$ & $2,7 \mathrm{a}$ & $2.178 \mathrm{c}$ & $101,4 \mathrm{~b}$ & $0,72 \mathrm{~b}$ & $0,73 \mathrm{a}$ \\
\hline L.29 & $\begin{array}{l}\text { Prostrado e de- } \\
\text { cumbente }\end{array}$ & $21,0 \mathrm{a}$ & $2,6 \mathrm{a}$ & $1.978 \mathrm{c}$ & $112,5 \mathrm{~b}$ & $0,67 \mathrm{~b}$ & $0,75 \mathrm{a}$ \\
\hline L.31 & Ereto e prostrado & $8,6 \mathrm{~b}$ & $1,8 \mathrm{~b}$ & $3.292 \mathrm{a}$ & $190,1 \mathrm{a}$ & $0,19 \mathrm{~d}$ & $0,36 \mathrm{~b}$ \\
\hline L. 37 & Prostrado & $9,7 \mathrm{~b}$ & $1,8 \mathrm{~b}$ & 906 e & $40,2 \mathrm{c}$ & $0,29 \mathrm{~d}$ & $0,11 \mathrm{~b}$ \\
\hline L. 38 & Ereto e prostrado & $8,5 \mathrm{~b}$ & $1,6 \mathrm{~b}$ & $1.003 \mathrm{e}$ & $58,9 \mathrm{c}$ & $0,80 \mathrm{a}$ & $0,47 \mathrm{~b}$ \\
\hline CV (\%) & -- & 26,2 & 9,1 & 15,4 & 18,4 & 13,1 & 32,6 \\
\hline
\end{tabular}

Valores seguidos pela mesma letra na coluna não diferem estatisticamente entre si, pelo teste Scott-Knott ao nível de 5\% (values followed by the same letter in the columns do not differ statistically by Scott-Knott test, $\mathrm{p}<0.05$ ). 
Tabela 2. Médias (\% relativa) dos principais componentes do óleo essencial de 16 acessos L. alba no Distrito Federal (main constituents of the essential oil of 16 accessions of L. alba in Distrito Federal). Brasília, Universidade de Brasília, 2006.

\begin{tabular}{lrrrrrr}
\hline \multirow{2}{*}{ Acessos } & \multicolumn{7}{c}{ Concentração relativa (\%) } \\
\cline { 2 - 7 } & Limoneno & Linalol & Mirceno & Geranial & Neral & Citral \\
\hline L.01 & $13,9 \mathrm{c}$ & $69,1 \mathrm{c}$ & $0,4 \mathrm{e}$ & $1,4 \mathrm{~b}$ & $1,2 \mathrm{c}$ & $2,6 \mathrm{c}$ \\
L.02 & $0,0 \mathrm{~d}$ & $4,0 \mathrm{~d}$ & $13,5 \mathrm{~b}$ & $29,7 \mathrm{a}$ & $23,5 \mathrm{a}$ & $53,3 \mathrm{a}$ \\
L.04 & $11,5 \mathrm{c}$ & $73,6 \mathrm{~b}$ & $0,4 \mathrm{e}$ & $0,7 \mathrm{~b}$ & $0,6 \mathrm{c}$ & $1,3 \mathrm{c}$ \\
L.05 & $0,0 \mathrm{~d}$ & $3,2 \mathrm{~d}$ & $15,7 \mathrm{~b}$ & $28,0 \mathrm{a}$ & $22,4 \mathrm{a}$ & $50,4 \mathrm{a}$ \\
L.16 & $0,0 \mathrm{~d}$ & $89,8 \mathrm{a}$ & $0,0 \mathrm{e}$ & $0,3 \mathrm{~b}$ & $0,3 \mathrm{c}$ & $0,6 \mathrm{c}$ \\
L.17 & $0,0 \mathrm{~d}$ & $3,1 \mathrm{~d}$ & $11,6 \mathrm{c}$ & $31,5 \mathrm{a}$ & $25,2 \mathrm{a}$ & $56,7 \mathrm{a}$ \\
L.20 & $0,9 \mathrm{~d}$ & $4,1 \mathrm{~d}$ & $8,8 \mathrm{c}$ & $23,2 \mathrm{a}$ & $18,6 \mathrm{~b}$ & $41,8 \mathrm{~b}$ \\
L.22 & $18,0 \mathrm{~b}$ & $5,8 \mathrm{~d}$ & $6,0 \mathrm{~d}$ & $24,7 \mathrm{a}$ & $19,7 \mathrm{~b}$ & $44,4 \mathrm{~b}$ \\
L.24 & $11,4 \mathrm{c}$ & $70,3 \mathrm{c}$ & $0,0 \mathrm{e}$ & $1,6 \mathrm{~b}$ & $1,3 \mathrm{c}$ & $2,9 \mathrm{c}$ \\
L.25 & $11,2 \mathrm{c}$ & $70,4 \mathrm{c}$ & $0,0 \mathrm{e}$ & $1,4 \mathrm{~b}$ & $1,2 \mathrm{c}$ & $2,6 \mathrm{c}$ \\
L.27* & $36,0 \mathrm{a}$ & $3,4 \mathrm{~d}$ & $0,9 \mathrm{e}$ & $0,0 \mathrm{~b}$ & $0,0 \mathrm{c}$ & $0,0 \mathrm{c}$ \\
L.28 & $10,8 \mathrm{c}$ & $72,7 \mathrm{~b}$ & $0,3 \mathrm{e}$ & $0,7 \mathrm{~b}$ & $0,6 \mathrm{c}$ & $1,3 \mathrm{c}$ \\
L.29 & $10,8 \mathrm{c}$ & $74,2 \mathrm{~b}$ & $0,4 \mathrm{e}$ & $0,9 \mathrm{~b}$ & $0,8 \mathrm{c}$ & $1,7 \mathrm{c}$ \\
L.31 & $0,2 \mathrm{~d}$ & $8,2 \mathrm{~d}$ & $6,0 \mathrm{~d}$ & $26,9 \mathrm{a}$ & $21,2 \mathrm{~b}$ & $48,1 \mathrm{~b}$ \\
L.37 & $2,4 \mathrm{~d}$ & $5,3 \mathrm{~d}$ & $47,6 \mathrm{a}$ & $0,6 \mathrm{~b}$ & $0,4 \mathrm{c}$ & $1,0 \mathrm{c}$ \\
L.38 & $22,6 \mathrm{~b}$ & $3,3 \mathrm{~d}$ & $0,6 \mathrm{e}$ & $28,2 \mathrm{a}$ & $22,8 \mathrm{a}$ & $51,0 \mathrm{a}$ \\
\hline CV (\%) & 32,53 & 5,98 & 31,37 & 23,46 & 18,70 & 20,85 \\
\hline
\end{tabular}

Valores seguidos pela mesma letra na coluna não diferem estatisticamente entre si, pelo teste Scott-Knott ao nível de $5 \%$ de probabilidade. *O acesso L.27 apresentou 46,9\% de carvona (values followed by same letter in the columns do not differ statistically by Scott-Knott test, $\mathrm{p}<0.05 .{ }^{*}$ Accession L. 27 showed $46,9 \%$ of carvone).

e L.31 (3.292 $\left.\mathrm{g} \mathrm{pl}^{-1}\right)$ apresentaram os maiores rendimentos de massa fresca não diferindo significativamente entre si, enquanto os acessos L.37 (906 g $\left.\mathrm{pl}^{-1}\right)$ e L.38 $\left(1.003 \mathrm{~g} \mathrm{pl}^{-1}\right)$ mostraram os menores rendimentos (Tabela 1).

A massa foliar seca variou de 207,7 $\mathrm{g} \mathrm{pl}^{-1}$ (L.22) a 40,2 $\mathrm{g} \mathrm{pl}^{-1}$ (L.37), com média $99,4 \mathrm{~g} \mathrm{pl}^{-1}$, destacando-se os acessos L.22 (207,7 $\left.\mathrm{g} \mathrm{pl}^{-1}\right)$ e L.31 (190,1 $\left.\mathrm{g} \mathrm{pl}^{-1}\right)$ que apresentaram os maiores rendimentos de massa seca, não diferindo significativamente entre si (Tabela 1).

Os acessos apresentaram teores médios do óleo essencial bastante variados. Os acessos L. 16 (0,84\%), L.38 (0,80\%) e L.24 (0,76\%) apresentaram as maiores concentrações de óleo essencial, não diferindo significativamente entre si. Considerando o rendimento de massa foliar seca por planta e o teor de óleo essencial de cada acesso, e calculando o rendimento de óleo por acesso $\left(\mathrm{g} \mathrm{pl}^{-1}\right)$ pode-se observar que os acessos mais produtivos ou com maior concentração de óleo essencial, não corresponderam àqueles com maior estimativa de rendimento por planta (Tabela 1). Este fato se deve à correlação negativa verificada entre estes parâmetros (Tabela 3). Os acessos L.1, L.4, L.16, L.24, L.25, L.28 e L.29 apresentaram rendimento de óleo por planta superior aos demais não diferindo significativamente entre si (Tabela 1).

O perfil aromático do óleo essencial dos 16 acessos avaliados apresentou composição química bastante heterogênea. Foram detectados em média 32 compostos no óleo essencial de cada acesso. Os constituintes majoritários foram: limoneno, linalol, mirceno, citral. Carvona foi identificada apenas no acesso L.27. O citral foi obtido pelo somatório dos teores de geranial e neral (Tabela 2).

O acesso L.27 (36\%) registrou o maior percentual relativo de limoneno seguido dos acessos L.38 (22,6\%) e L.22 $(18,0 \%)$, que não diferiram significativamente entre si. Os acessos L.01; L.04; L.24; L.25; L.28 e L.29 apresentaram teores de limoneno que variaram de $13,9 \%$ a $10,8 \%$, não diferindo significativamente entre si. Não foi detectada a presença de limoneno nos acessos L.02; L.05; L.16 e L.17.

Os acessos L.01, L.04, L.16, L.24,
L.25, L.28 e L.29 apresentaram concentrações de linalol superiores à média (35,03\%) destacando-se o acesso L.16 $(89,8 \%)$ que mostrou concentração significativamente superior aos demais acessos e aos valores relatados por Tavares et al. (2005), de $65,1 \%$ a $74,0 \%$ e Lorenzo et al. (2001) de 55,3\%.

O acesso L.37 (47,6\%) apresentou teor de mirceno significativamente superior aos demais, correspondendo a 6,7 vezes o valor da média. Os acessos L. 2 (53,3\%), L.5 (50,4\%), L. 17 (56,7\%) e L.38 (51,0\%) apresentaram os maiores teores de citral, não diferindo significativamente entre si. O acesso L.27 (46,9\%) foi o único a apresentar carvona.

A área foliar correlacionou-se positivamente com o comprimento da haste $\left(0,656^{* *}\right.$, moderada), teor do óleo essencial $\left(0,374^{* *}\right.$, fraca) e concentração de linalol $\left(0,782^{* *}\right.$, forte) e negativamente com a concentração dos demais componentes do óleo (Tabela 3).

O comprimento da haste correlacionou-se positivamente com o teor de óleo $\left(0,426^{* *}\right.$, moderada $)$ e a concentração de linalol $\left(0,752^{* *}\right.$, forte) e negativamente com os demais componentes do 
Tabela 3. Coeficientes de correlação de Pearson entre as variáveis analisadas de 16 acessos de L. alba no Distrito Federal (Person's correlation coeficient among analyzed variables of 16 accessions of $L$. alba from Distrito Federal). Brasília, Universidade de Brasília, 2006.

\begin{tabular}{|c|c|c|c|c|c|c|c|c|c|c|}
\hline Variáveis & 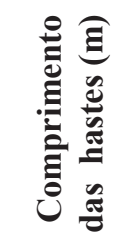 & 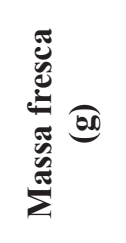 & 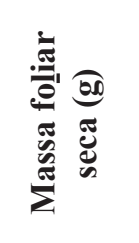 & 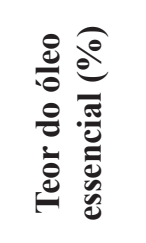 & 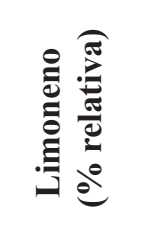 & 可 & 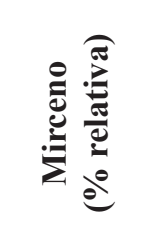 & فْ & 苞 & 㺼 \\
\hline Área foliar $\left(\mathrm{cm}^{2}\right)$ & $0,656^{* *}$ & $-0,046^{\mathrm{ns}}$ & $0,015^{\mathrm{ns}}$ & $0,374 * *$ & $-0,148^{\mathrm{ns}}$ & $0,782^{* *}$ & $-0,332 *$ & $-0,494 * *$ & $-0,495^{* *}$ & $-0,494 * *$ \\
\hline $\begin{array}{l}\text { Comprimento das hastes } \\
\text { (m) }\end{array}$ & & $-0,018^{\mathrm{ns}}$ & $0,019^{\mathrm{ns}}$ & $0,426^{* *}$ & $0,067^{\text {ns }}$ & $0,752^{* *}$ & $-0,351 * *$ & $-0,591 * *$ & $-0,592 * *$ & $-0,591 * *$ \\
\hline Massa fresca $(\mathrm{g})$ & & & $0,851 * *$ & $-0,551 * *$ & $-0,195^{\mathrm{ns}}$ & $-0,148^{\mathrm{ns}}$ & $-0,183^{\text {ns }}$ & $0,409 * *$ & $0,405 * *$ & $0,407 * *$ \\
\hline Massa foliar seca $(\mathrm{g})$ & & & & $-0,444 * *$ & $-0,002^{\mathrm{ns}}$ & $-0,083^{\mathrm{ns}}$ & $-0,243 *$ & $0,286^{*}$ & $0,282^{*}$ & $0,284^{*}$ \\
\hline Teor do óleo essencial (\%) & & & & & $0,411 * *$ & $0,652 * *$ & $-0,538 * *$ & $-0,573 * *$ & $-0,568 * *$ & $-0,571 * *$ \\
\hline Limoneno (\% relativa) & & & & & & $0,011^{\mathrm{ns}}$ & $-0,409 * *$ & $-0,325^{*}$ & $-0,322^{*}$ & $-0,324 *$ \\
\hline Linalol (\% relativa) & & & & & & & $-0,499 * *$ & $-0,747 * *$ & $-0,746^{* *}$ & $-0,747 * *$ \\
\hline Mirceno (\% relativa) & & & & & & & & $0,161^{\mathrm{ns}}$ & $0,156^{\mathrm{ns}}$ & $0,159^{\text {ns }}$ \\
\hline Geranial (\% relativa) & & & & & & & & & $0,999 * *$ & $0,999 * *$ \\
\hline Neral (\% relativa) & & & & & & & & & & $0,999 * *$ \\
\hline
\end{tabular}

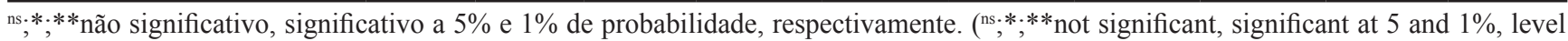
of probability, respectively).

óleo. Os acessos que apresentaram o linalol como um dos principais constituintes, tenderam a apresentar maior área foliar, comprimento das hastes e teor de óleo essencial.

A massa fresca da planta correlacionou-se positivamente com a massa seca das folhas $\left(0,851^{* *}\right.$, forte $)$ e negativamente com o teor de óleo essencial $\left(-0,551^{* *}\right.$, moderada). A massa da folha seca correlacionou-se negativamente com o teor de óleo essencial $\left(-0,444^{* *}\right.$, moderada), (Tabela 3 ) indicando que os acessos com maior produção de massa foliar não correspondem àqueles com maiores teores e rendimento de óleo essencial, provavelmente, pelo efeito de diluição ou por apresentarem um número menor de glândulas oleíferas.

O teor de óleo essencial correlacionou-se positivamente com a concentração de limoneno $\left(0,411^{* *}\right.$, moderada $)$ e linalol $\left(0,652^{* *}\right.$, forte $)$ e negativamente com os demais parâmetros, consequentemente os acessos com maior concentração de óleo essencial mostram a tendência para a maior produção de limoneno e linalol.

Considerando os compostos majoritários com teores acima de $10 \%$ foram identificados os seguintes quimiotipos: citral (L.20 e L.31); citral-limoneno (L.22 e L.38); citral-mirceno (L.2, L.5 e L.17), mirceno (L.37), limonenocarvona (L.27), linalol (L.16), linalollimoneno (L.1, L.4, L.24, L.25, L.28 e L.29).

Relacionando-se o rendimento de óleo essencial (Tabela 1) com a concentração relativa dos principais componentes do óleo dos 16 acessos (Tabela 2) observa-se que o acesso L.16 (0,77 $\mathrm{g} \mathrm{pl}^{-1}$ ) destacou-se como fonte potencial para a produção de linalol; o acesso L.17 $\left(0,17 \mathrm{~g} \mathrm{pl}^{-1}\right)$ como fonte de mirceno; o acesso L.27 como fonte de limoneno $\left(0,17 \mathrm{~g} \mathrm{pl}^{-1}\right)$ e de carvona $\left(0,21 \mathrm{~g} \mathrm{pl}^{-1}\right) \mathrm{e}$ o acesso L.38 $\left(0,24 \mathrm{~g} \mathrm{pl}^{-1}\right)$ como fonte de citral.

Os dezesseis acessos de L. alba estudados apresentaram variações fenotípicas e diferentes constituintes químicos no óleo essencial demonstrando a grande variabilidade genética, característica de plantas nativas ainda não domesticadas. Esta variabilidade pode ser considerada favorável nos programas de melhoramento, pois oferece suporte para seleção de acessos superiores direcionados às diversas demandas de utilização dessa espécie. Por outro lado, esta variabilidade de tipos químicos pode ser considerada preocupante do ponto de vista de utilização da planta como fitoterápico, pois o usuário poderá estar utilizando material não adequado para atingir o objetivo desejado. Esse fato é muito comum no Brasil envolvendo várias espécies de plantas medicinais. Desta forma, para atender este seguimento, sugere-se programas de distribuição de mudas com identificação botânica e química dos acessos L. alba.

\section{REFERÊNCIAS}

ALEAJAP; ORTEGALAG; ROSADO PEREZA; JORGE MR; BALUJA R. 1997. Composición y propiedades antibacterianas del aceite esencial de Lippia alba (Mill.) N. E. Br. Revista Cubana Farmácia 30: 1-6.

CASTRO DM. 2001. Efeitos da variação sazonal, colheita selecionada e diferentes temperaturas de secagem sobre a produção de biomassa, rendimento e composição de óleos essenciais de Lippia alba (Mill.) N.E.Br. ex. Britt e Wilson (Verbenaceae). Botucatu: UNESPFCA. 132p (Tese doutorado).

EHLERT PAD. 2003. Épocas de plantio, idade e horário de colheita na produção e qualidade do óleo essencial de quimiotipo limoneno-carvona de Lippia alba (Mill.) N.E. Br. Botucatu: UNESP-FCA. 107p (Tese doutorado).

GILBERT B; FERREIRA JLP; ALVES LF. 2005. Monografias de plantas medicinais brasileiras e aclimatadas. Curitiba: Abifito. 250p.

LORENZI H; MATOS FJA. 2002. Plantas medicinais no Brasil: Nativas e exóticas cultivadas. Nova Odessa: Instituto Plantarum. $512 \mathrm{p}$.

LORENZO D; PAZ D; DAVIES P; VILA R; CANIGUERAL S; DELLACASSA E. 2001. Composition of a new essential oil 
type of Lippia alba (Mill.) N.E.Brown from Uruguay. Flavour and Fragrance Journal 16: 356-359.

MATOS FJA. 1996. As ervas cidreiras do Nordeste do Brasil - Estudo de três quimiotipos de Lippia alba (Mill.) N.E Brown (Verbenaceae). Parte I - Farmacognosia. Revista Brasileira de Farmácia 77: 65-67.

PEREIRA PINTO JEB; SANTIAGO EJA; LAMEIRA O. 2000. Compêndio de plantas medicinais. Lavras: UFLA/FAEPE. 208 p.

PESSINI GL; HOLETZ FB; SANCHES NR; CORTEZ DAG; DIAS FILHO BP; NAKAMURA CV. 2003. Avaliação de atividade antibacteriana e antifúngica de extratos de plantas utilizadas na medicina popular. Revista Brasileira de Farmacognosia
13: 21-24.

POTZERNHEIM ML; COSTA AF; BIZZO HR; CARVALHO SILVA M; VIEIRA RF. 2006.

Essential Oil of Piper xylosteoides (Kunth) Steud. from Federal District, Brazil. Journal of Essential Oil Research 18: 523-524.

SHIMAKURA SE; RIBEIRO JUNIOR PJ. 2006. Estatística. Disponível em www.est.ufpr. br/ paulojus/CE003/ce003.html. Acessado em 10 de julho de 2006.

SIMÕES CMO; SPITZER V. 2003. Óleos voláteis. In: SIMÕES CMO; SCHENKEL EP; GOSMANN G; MELLO JCP; MENTZ LA; PETROVICK PR. (org). Farmacognosia: da planta ao medicamento; $5^{\circ}$ ed. Porto Alegre/ Florianópolis: UFRGS/UFSC. p. 467-495.

SOARES LS. 2001. Estudo tecnológico, fotoquímico e biológico de Lippia alba (Mill.) N. E. Brown ex Britt.\&Wils. (Falsa-melissa) Verbenácea. Florianópolis: UFSC. 182 p (Tese doutorado).

STASHENKO EE; JARAMILHO BE; MARTINEZ JR. 2003. Comparacion de la composicion química y de la actividad in vitro de los metabolitos secundários volátiles de plantas de la família Verbenáceas. Revista de la Academia Colombiana de Ciencias, Exactas, Físicas y Naturales 27: 579-597.

TAVARES ES; JULIÃO LS; LOPES D; BIZZO HR; LAGE CLS; LEITÃO SG. 2005. Análise do óleo essencial de folhas de três quimiotipos de Lippia alba (Mill.) N.E.Br. (Verbenácea) cultivados em condições semelhantes. Revista Brasileira de Farmacognosia 15: 1-5. 